Research Article

\title{
Mathematical Modelling of the Linear Friction Welding Process
}

\author{
A.Vairis* \\ Dpt of Mechanical Engineering, TEI of Crete, Heraklion, Greece
}

Received 15 September 2012; Accepted 28 September 2012

\begin{abstract}
Modelling of complex industrial processes such as linear friction welding can be addressed with the aid of various mathematical techniques to investigate individual process phases. The initial frictioning phase can be studied with analytical contact models to identify different frictional regimes and relate them to actual friction coefficient experiments, which in turn will be used in process parameters selection and numerical modelling. The use of ANNs for the same phase can help in the prediction of the coefficient of friction as well. For the steady state extrusion phase of the process material flow analytical models may predict the strain rates that material experiences at and near the welding interface, which directly influences localized yield and subsequent plastic material flow. Analytical models can also assist in the correct selection of process parameters by modeling material flow as a thin lubricating film.
\end{abstract}

Keywords: Linear friction welding, analytical modeling, friction, material extrusion, Ti6Al4V

\section{Introduction}

Science employs a number of diverse methods and techniques for modeling physical and industrial processes alike. Industrial processes are often associated with a number of problems, which mostly result from the lack of understanding of the key physical properties and characteristics of either the materials involved or the manufacturing processes themselves. These problems often impact on end-product quality, as well as production efficiency. In industrial practice, the solution to these problems is traditionally largely based on empiricism. While it is possible to arrive at an acceptable practical solution using such an approach, it lacks generality and does not provide a really good understanding of the causes of the problems. Today, rapid advances in computational science and resources have enabled the development of simulation tools which help in identifying the sources of these problems, providing process optimization solutions and, where possible, completely designing out of the problems [1].

\section{The linear friction welding process}

Linear friction welding is a solid state process for joining materials together through intimate contact of a plasticised interface, which is generated by frictional heat produced as one component is moved under pressure in a direct reciprocating mode relative to another. It is a relatively new process, which began to be investigated in the 1980s and soon after the first commercial machines appeared to join aircraft titanium alloy components. This joining process for

\footnotetext{
*E-mail address: vairis@staff.teicrete.gr

ISSN: 1791-2377 @ 2012 Kavala Institute of Technology. All rights reserved.
}

metals is observed to have distinct phases [2] and are presented here for completeness.

Phase I, The Initial Phase. From the beginning the two workpieces are moving under pressure in a linear reciprocating manner. Heat is generated at the rubbing interface due to solid friction. True surface contact area increases throughout this phase due to wear and the thermal softening effects of movement. No axial shortening of the specimens is experienced at this stage. This phase is critical for the rest of process to proceed, for if insufficient heat is generated the next phase will not follow.

Phase II, The Transition Phase. During this very short period, the heat affected zone expands from the asperities into the bulk of the material until phase III is reached. The true contact area is considered to be $100 \%$ of the cross sectional area due to wear and localised yielding of the asperities. A plasticised layer is formed between the two rubbing surfaces which cannot support the axial load, thus deforming permanently.

Phase III, The Equilibrium Phase. Axial shortening begins to register as plasticised matter is expelled into an upset. Material in the heat affected zone that has yielded moves out of the rubbing interface aided by oscillatory movement. This forms a flash, which may take different shape and size depending on the material extruded. Melting conditions at the interface are not reached as experimental data have shown [3]. The phase is termed "equilibrium" to reflect the fact that as long as oscillation occurs axial shortening increases while not leading automatically to another phase.

Phase IV, The Deceleration/Forging Phase. When the desired upset is reached the two materials are brought to rest very rapidly and a forging pressure may be applied to consolidate the weld. 
The process is self regulating as the appropriate choice of process parameters (amplitude of oscillation, frequency of oscillation, friction pressure) leads to phase III, which is necessary to be present to form sound welds. Depending on the machine used the process is controlled by the length of axial shortening achieved or process time, producing with excellent repeatability good joints. Joining may occur at a different phase of the process (III or IV) depending on the material.

\section{Modelling the beginning of the process}

The movement of an object along a surface is resisted by forces commonly referred to as friction. These are nonconservative and convert kinetic energy of moving objects into thermal or mechanical energy, as evident by the increase in temperature at the rubbing interface or squeaking noises. In most cases the precise value of the coefficient of friction depends strongly on the conditions under which it is measured. To successfully model manufacturing processes where friction plays an important role, such as friction welding, it is necessary to establish the friction characteristics of the materials involved under conditions that typify the system under study.

\subsection{Frictional behaviour}

With reference to the modeling needs of LFW a study was made [4] where a number of measurements were made under different sliding conditions for different interface temperatures in an experimental setup to measure the coefficient of friction. Figure 1 shows the relationship between the coefficient of friction and interface temperature for various rubbing velocities for the titanium alloy Ti6Al4V. The alloy studied showed sensitivity to rubbing velocity $(480 \mathrm{~mm} / \mathrm{s})$. This can be attributed to stick-slip behaviour, as the analytical model shown later suggests. Increasing sliding speed has a direct beneficial effect on thermal softening. To verify the applicability of the procedure followed, intermediate values of friction coefficient were compared to predicted values of the coefficient. The recorded values were within $10 \%$ of the values expected by curves fitted to experimental data.

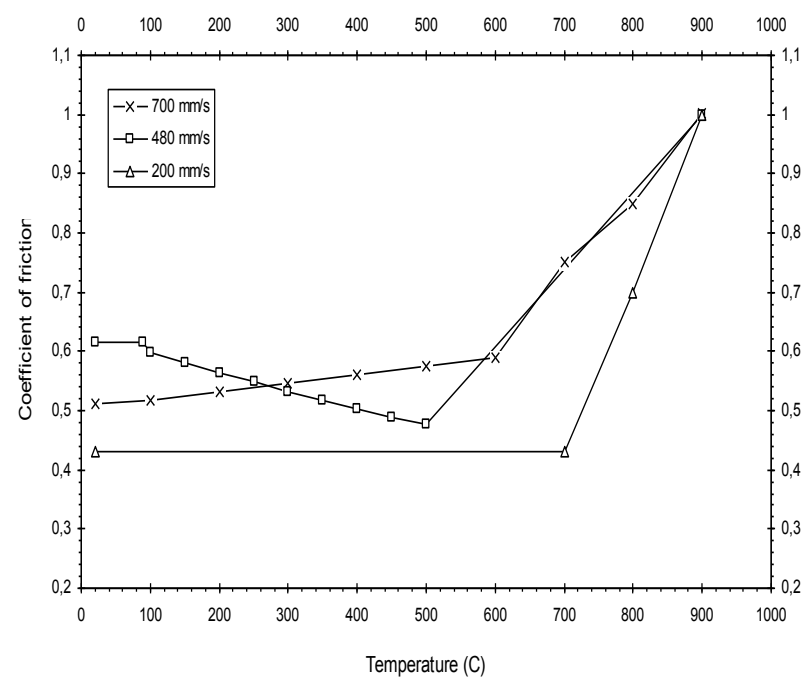

Fig. 1. Variation of friction coefficient with temperature at different sliding velocities for Ti6A14V [3].

\subsection{Analytical model of frictional movement}

Among the various modeling studies on frictional phenomena presented in literature, a contact model has been utilized to model the sliding motion of two plates by relating microscopic phenomena to macroscopic motion of a top plate relative to a stationary bottom plate [5]. These rigid surfaces are connected by junctions that spontaneously break and form upon contact, which behave as elastic springs. A spring of constant $\mathrm{K}$ is exerting the necessary force to move the top surface at a constant velocity $\mathrm{V}$. The equation of motion is as follows:

$$
M \ddot{X}+n \dot{X}+F_{b}+K(X-V t)=0
$$

where the force due to the interaction between the junctions and the driven surface is $F_{b}$, and $\eta$ is the damping coefficient. Coefficients $K$ and $\eta$ are material properties. The force $F_{b}$ is given by the equation:

$$
F_{b}=\sum_{i=1}^{N} q_{i} f_{i}^{(x)}
$$

where $q_{i}$ indicates the state of the junction ( 1 for formed, 0 for breached junction).

To calculate the elastic force $f_{i} l_{i}$ is the length of the junction and $l^{(0)}$ the distance between the plates:

$$
f_{i}=\kappa\left[l_{i}-l^{(0)}\right]
$$

The top surface continuously forms junctions that hinder sliding and extend and breaks them. Each junction produces different elastic forces. The state $q_{i}$ of each junction can be described:

$$
\begin{aligned}
& q_{i}(t+\Delta t)=q_{i}(t)-q_{i}(t) \theta\left(\xi_{i}-\Delta t k_{o f f}\right)+ \\
& {\left[1-q_{i}(t)\right] \theta\left(\xi_{i}-\Delta t k_{o n}\right)}
\end{aligned}
$$

where $\Delta t$ is the time step, $\xi_{i}$ is a random variable in the interval $(0,1)$ and $\theta(z)$ is the Heaviside step function for the description of the stochastic fracture (creation) of a junction that occurs for $\xi_{i}>\Delta t k_{\text {off(on) }}$. The rate of creation and fracture of junctions is $k_{\text {on }}$ and $k_{\text {off }}$, respectively.

Junctions can be of two types: weak or strong junctions depending on their energy being slightly larger or much larger than $k_{B} T$, respectively. Junction creation is characterized by the rate $k_{o n}$, which is assumed to depend only on the age of the contact $\tau$ :

$$
k_{\text {on }}=k_{\text {on }}^{0} g\left(\tau, \tau_{0}\right)
$$

with $k_{o n}^{0}$ being the rate of junction creation for a stationary contact, $\tau_{0}$ some critical junction age and $g$ a modified stepwise function. Contact time $\tau$ is related to sliding velocity and the typical length scale of the contact $\alpha$ through the equation $\tau=\alpha / \dot{X}$. The characteristic time-scale $\tau_{0}$ necessary for junction creation, is used to define a critical velocity $V_{0}=\alpha / \tau_{0}$. Above this, velocity junctions cannot be formed as in stick-slip. 
In Figure 2, the temporal evolution of the friction force is depicted, where it was found that equilibrium was attained after a few steps, which is in agreement with theoretical [6] and experimental observations [3]. Then, varying the velocity, predictions were obtained for friction force where two sliding regimes (low and high velocities) with a stickslip region between them were identified.

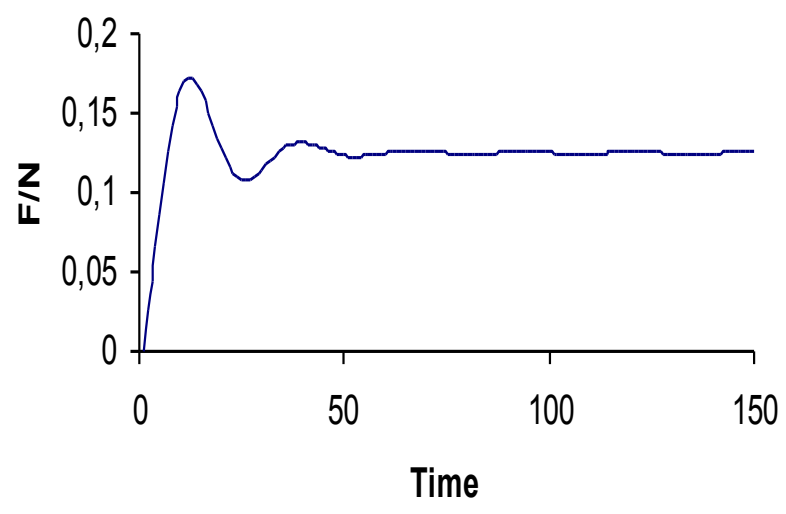

Fig. 2. Temporal variation of friction force, as calculated by the model for parameter values $\mathrm{V}=0.5, \mathrm{~N}=300, \mathrm{~K}=150.0, \quad \eta=75.0, \kappa=\lambda=0.1$, $1(0)=\alpha=\Delta x=1.0, \mathrm{k} 0=\mathrm{k} 0$ on $=0.1, \tau 0=2.0, \Delta \mathrm{t}=1.0$.

The three frictional regimes exhibit different behaviour, with different mechanisms being responsible for them. The low sliding velocity regime represents a state where thermal bond dissociation determines junction fracture rather than shear-induced stress. This regime behaviour correlates with the observation that the value of static friction depends on time scale. This region is consistent with atomic scale stickslip motion of individual junctions. The low sliding velocity frictional regime, which is characterised by energy dissipation, is dependent on the fracture and the subsequent relaxation of junctions, rather than on viscous dissipation.

In the stick-slip region, at intermediate sliding velocities, the processes of spontaneous and shear-induced bond dissociation compete to produce an erratic stick-slip motion. Junction fracture is controlled by the effect of shear stress on activation barrier. At this time the fraction of intact junctions decreases with a similar decrease of the fracture contribution to the energy dissipation. The net kinetic friction is relatively insensitive to sliding velocity as the effect of the diminishing fractures contribution is counterbalanced by the viscous component of energy dissipation. The system shows a cooperative behaviour, where as the number of breaking bonds increases the force on the remaining bonds increases and bond rupture synchronises, thus producing a more consistent stick-slip behaviour. There is a correlation between macroscopic frictional properties and a collective behaviour of microscopic bonds [7].

The high sliding velocity region shows a transition from stick-slip behaviour to smooth sliding, as bond formation becomes impossible due to short contact times. Frictional force in this regime is completely determined by viscous dissipation.

The results of these simulation are in qualitative agreement with experimental data available for titanium alloy Ti6A14V (Fig.1, [3]), where it can be seen that for intermediate sliding velocities friction force decreases with temperature while for low and high sliding velocities increases.

\subsection{Artificial intelligence model}

Other studies have focused on modelling the frictional process by artificial intelligence with a symbolic or qualitative description. Artificial Neural Networks have been employed using experimental data of composites and coated materials [8], [9], [10], [11] which have been employed to produce with reasonable accuracy predictions of friction coefficient and with limited success of wear.

Using sets from the same frictional behaviour experiments as a training sample two different architectures of ANN were trained [12]. In this way, the ability and generalization capability of the proposed ANN was evaluated. All input and output data were normalized.

(1) Multi Layer Feed Forward Network Model

A multi-layered feed-forward (MLFF) neural network with 4 layers was tested. The input layer had three neurons which were fed in with input variables (velocity, temperature, normal stress). The output neuron is used to estimate friction coefficient $\mu$. The standard back propagation algorithm with momentum as proposed in [13] was used to train the network. The gradient descent method was also used to search for the optimal weights settings. The weight updating method chosen was the delta learning rule. In the model an accumulation of weight changes over training sessions is used to eliminate the structural presentation of the training set problem.

Although one hidden layer is capable of modelling any function [14], [15], two hidden layers were used for a more compact architecture and learning efficiency with backpropagation. Full connectivity between adjacent layer nodes was used. After testing the optimal size was determined to be a network of 10 and 6 neurons (i.e. $3 \times 10 \times 6 \times 1$ ) in the 1 st and 2 nd hidden layer respectively. Better generalization and faster convergence was achieved with symmetric functions with the bipolar sigmoidal transfer function (for the hidden neurons) exhibiting the best behaviour

$$
y_{i}=\frac{2}{1+e^{-\beta x_{i}}}-1
$$

where $\mathrm{xi}$ is the input and yi is the output for the ith hidden layer neuron. Coefficient $\beta$ which controls the steepness of the function curve was set to 0.2 . Two other parameters were used for rapid learning: the momentum term $\alpha$ and the learning rate $\eta$ with $\eta=0.9$ and $\alpha=0.6$ set at the beginning of training and towards the end were reduced gradually to $\eta=0.3$ and $\alpha=0.1$.

\section{(2.) Radial Basis Function Architecture Model}

The RBF type ANN was also studied, which although requiring more neurons, its design and training time was shorter. RBF networks exhibit better performance especially for many training vectors. This type of network utilizes Gaussian functions for activation and the Euclidean distance weight functions from its input, while the training algorithm alters dynamically the neurons number until the desirable result is achieved. The hidden layer neurons have a nonlinear activation function while the output layer neurons a linear one. The training procedure is similar to MLFF ones, with the exception that the maximum number of neurons is determined.

For both ANNs models performance was assessed using three different parameters. The first one was the mean 
squared normalized error (MSE_N) which is given by,

$$
M S E_{-} \mathrm{N}=\frac{1}{P} \int_{i=1}^{P} e_{i}^{2} \cdot d t
$$

where $\mathrm{P}$ is the number of data patterns, $\mathrm{e}$ is the error between the target and the ANN's predicted value (ei=ti-oi). The second parameter was the mean absolute error (MAE)

$$
M \mathrm{~A} E=\frac{1}{N} \int_{i=1}^{N}\left|e_{i}\right| \cdot d t
$$

and the third one is the mean squared error with regularization (MSE-Reg) which measures network performance as the weighted sum of the mean squared error and the mean squared weight and bias values,

$$
M S E \_\operatorname{Re} g=\gamma M S E+(1-\gamma) \frac{1}{N} \sum_{j=}^{N} w_{j}^{2}
$$

where $\gamma$ is the performance ratio and wj the relevant neurons weight.

It was clearly shown that an appropriate ANN model architecture can be employed effectively for friction coefficient estimation (see Fig.3). Furthermore, the larger the training and testing data set there is the more accurate results are achieved and the more robust is the ANN performance.

\subsection{Analytical heat flow model}

An analytical model has been developed [16], which may give some insight into the early stages of linear friction welding. In order to retain a certain degree of predictive ability, this model forms a physical framework within which the semi-empirical descriptions of the material behaviour are incorporated. It is assumed that at the beginning of the motion the two specimens are resting on asperities at the interface. These deform predominantly in a plastic fashion under the normal load applied, as the contact is between rough metal surfaces. This incipient yield condition means that the area of contact will depend on the applied normal load and the yield stress of the material, with the latter depending on temperature. As temperature increases due to friction, a reduction in yield stress will occur, as well as an increase in the area of contact. Thus the shear force will, under certain conditions, remain constant during phase I.

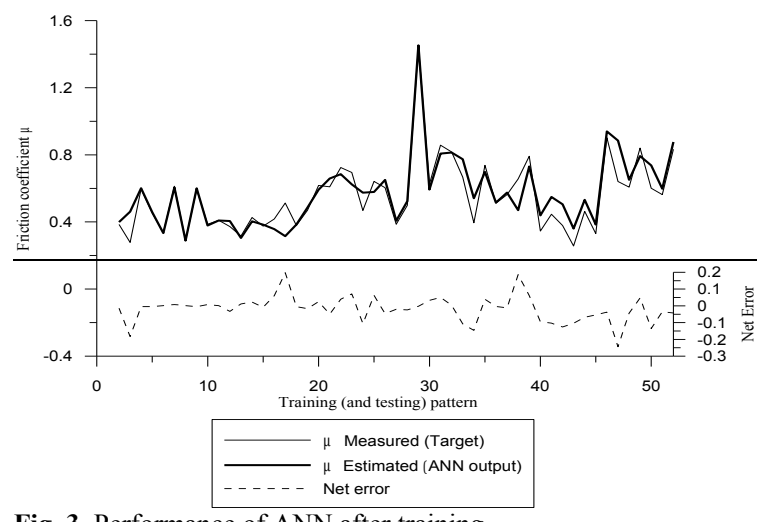

Fig. 3. Performance of ANN after training
The specimens move in a sinusoidal motion. The shear stress $(\tau)$ exerted on the interface being dependent on $\mu$ the coefficient of friction and $\mathrm{P}_{\mathrm{N}}$ the normal pressure. The heat generation rate per cycle per unit interfacial area (q) is

$\mathrm{q}=\mu \mathrm{P}_{\mathrm{N}} v$

The average heat rate can be determined by integrating over a cycle

$q_{0}=\frac{1}{T} \int_{0}^{T} q d t=\frac{4}{T} \int_{0}^{T / 4} q d t=\frac{4}{T} \int_{0}^{T / 4} \mu P_{N} v d t=$

$=\frac{4}{T} \int_{0}^{T / 4} \mu P_{N} \alpha \omega \cos (\omega t) d t=\frac{2}{\pi} \mu P_{N} \alpha \omega$

This static model is similar to the case of a heat flux input to a solid (slab) bounded by a pair of parallel planes, one of which is at the rubbing interface, which has been solved analytically, with the heat flux a prescribed function of time. The total heat flux is assumed to be a prescribed function of time, as it is related to the increase of true area of contact during initial rubbing. The true contact area is assumed to increase linearly from zero to the cross sectional area of the specimens. No heat losses are assumed to the environment, as phase I is very short taking only a few seconds. Temperature as a function of time during phase I will be [5]

$$
\begin{aligned}
& \partial=\frac{2^{m+1} q_{0} k 1 / 2 t^{(m+1) / 2} \Gamma\left({ }_{2}^{1} m+1\right)}{K} * \\
& * \sum_{n=0}^{\infty}\left\{i^{m+1} \operatorname{erfc} \frac{(2 n+1) L_{0}-x}{2(k t)^{1 / 2}}+i^{m+1} \operatorname{erfc} \frac{(2 n+1) L_{0}+x}{2(k t)^{1 / 2}}\right\}
\end{aligned}
$$

where $\kappa$ is the diffusivity, $\Gamma$ the Euler gamma function, $t$ the time, Lo the length (overhang of the specimen from specimen holding bracket) of the specimen. The flux input is a prescribed function of time $q_{0} t^{1 / 2 m}$, where $\mathrm{m}=-1,0,1$. In this case $m=2$ as the flux is assumed to change linearly with time as is the true area of contact.

Eq. (12) predicts that heating time will be inversely proportional to the square of the ratio of the heat generation per unit interfacial area. As a result of that, short heating time requires the use of high specific power during welding, which can be achieved with high rubbing velocities, small areas of contact, high friction pressures or oscillating conditions which favour high coefficients of friction, depending on the material.

This model was used to predict temperature profiles during initial rubbing with non-linear material properties where the yield stress of Ti6Al4V assumed to change linearly with temperature. Other mechanical and physical properties were taken at average values for this temperature range. The true area of contact was taken to change linearly with time as well as the friction coefficient.

Experimentally measured temperatures were compared to calculated for oscillating Ti6Al4V specimens at a frequency of $30 \mathrm{~Hz}$ with an interface area of $60 \mathrm{~mm}^{2}$ and amplitude of oscillation of $0.92 \mathrm{~mm}$. From experimental data the time length of phase I was identified to be 4 seconds 
approximately. The temperature profile predicted is similar in shape to the experiment, with the temperature rising rapidly at the beginning and then decreasing its rate of increase and reaching a higher temperature at the end of the phase. The importance of the temperature dependent material properties is shown when temperature independent properties were used (see Fig.4).

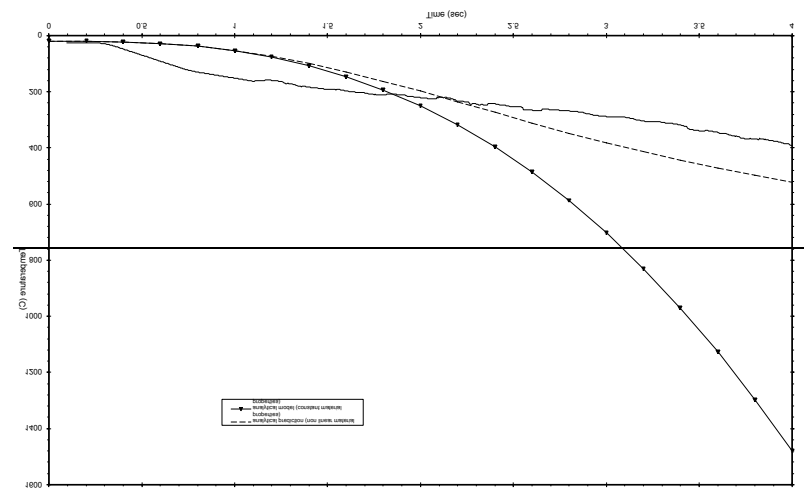

Fig. 4. Comparison between experimental and analytical model temperature data for initial phase

\section{Modelling the extrusion phase of the process}

\subsection{Material flow model}

During extrusion in phase III strain rates encountered by the rubbing material must be associated to the amplitude and frequency of oscillation, as well as the size of the heat affected zone formed at the interface. These high strain rates will be present in a narrow zone adjacent to the rubbing interface and will unavoidably affect yielding of hot material. If strain rates are high enough to limit the drop in yield stress of the material due to temperature, plasticity will not appear and material extrusion will not take place or be uniform.

It is assumed that during this phase steady state conditions have been reached, with the rate of axial shortening being effectively constant. It is supposed that the size or shape of these regions does not change with time and that the elastic section of the specimen supports the sections that have plastically deformed. Another simplification allowed is the assumption that material extrudes continuously during movement.

The distribution of strain rate can be calculated from a number of velocity equations [2]. The Cartesian velocity profiles along the interface are assumed to follow the profile of the deformed sections. Material extrusion is assumed to be due to the combined effect of friction pressure, which extrudes material from all faces of the specimen, and oscillatory movement. The latter is assumed to affect the velocity profile in the direction of movement. The following kinematically admissible velocity equations in the Cartesian directions can be formulated:

$$
v_{x}=v_{z}+v\left[1-\left(\frac{y}{y_{p l}}\right)^{\xi}\right], \quad y \leq y_{p l}
$$

or

$$
v_{x}=\beta v_{0} \frac{x}{L / 2}\left[1-\left(\frac{y}{h}\right)^{\phi}\right]+v\left[1-\left(\frac{y}{y_{p l}}\right)^{\xi}\right]
$$

$$
v_{\mathrm{y}}=v_{\mathrm{y}}(\mathrm{y})
$$

$v_{z}=\beta v_{0} \frac{z}{W / 2}\left[1-\left(\frac{y}{h}\right)^{\phi}\right], \quad y \leq h$

where $\mathrm{z}$ is the direction perpendicular to movement, $\mathrm{x}$ the direction of movement and $y$ the axial distance in the heat affected zone from the interface; $v_{0}$ the axial shortening velocity, $\mathrm{y}_{\mathrm{pl}}$ the width of the plastic zone, $\mathrm{h}$ the total width of the plastic zone. The term $\xi$ is a dimensionless term which characterises the strain rate distribution in the plastic section, $\varphi$ another dimensionless term characterising the flash shape and $\beta$ a dimensionless term characterising the velocity distribution in the plastic zone. Eq.(13) for $v_{z}$ suggests that it increases from zero at the border of the undeformed material to a maximum value of $v_{z}=\beta v_{0} 2 z / w$ at the rubbing interface

The dimensionless term $\rho_{\mathrm{s}}$ can be evaluated by measuring the length $(\ell)$ of the extruded matter at $y=0$. The flash is generated during axial shortening time $\left(t_{\text {axial }}\right)$, which is less than the total welding time. Then $\ell$ can be calculated by integrating the velocity component in the direction perpendicular to movement $v_{z}$ over $t_{\text {axial }}$ :

$l=\int_{0}^{t_{a x i a l}} v_{z}(y=0) d t=\frac{\phi+1}{\phi} \cdot \frac{v_{0} z t_{\text {axial }}}{h W\left(\frac{1}{L}+\frac{1}{W}\right)}$

estimating it at the face of the specimen, i.e. $z=\frac{W}{2}$

then

$$
\rho_{s}=\frac{v_{o} t_{\text {axial }}}{2 h l\left(\frac{1}{L}+\frac{1}{W}\right)-v_{o} t_{\text {axial }}}
$$

The application of this model to LFW requires the estimation of $\xi$. This parameter influences the form of the velocity field in the direction of oscillation. Its value can be estimated at the centre of the specimens by the difference of the velocity components:

$$
\xi=\frac{\ln \left(1-\frac{v_{x}-v_{z}}{v}\right)}{\ln \left(\frac{y}{y_{p l}}\right)}
$$

Using these equations, the main components of strain rate were calculated for two experiments done at $0.92 \mathrm{~mm}$ amplitude of oscillation and frequencies of $65 \mathrm{~Hz}$ and 100 $\mathrm{Hz}$ (see Fig.5 for prediction of strain rates in the $\mathrm{xx}$ and $\mathrm{zz}$ direction). Using this analytical model it is indicated that higher strain rates are encountered at the interface with increasing frequency of oscillation. For the rubbing interface to reach yielding conditions increased friction pressures will be required as the material in study (Ti6A14V) is strain rate 
sensitive. This increased requirement of input power with increasing frequency of oscillation had been observed in experiments up to $119 \mathrm{~Hz}$ [3].

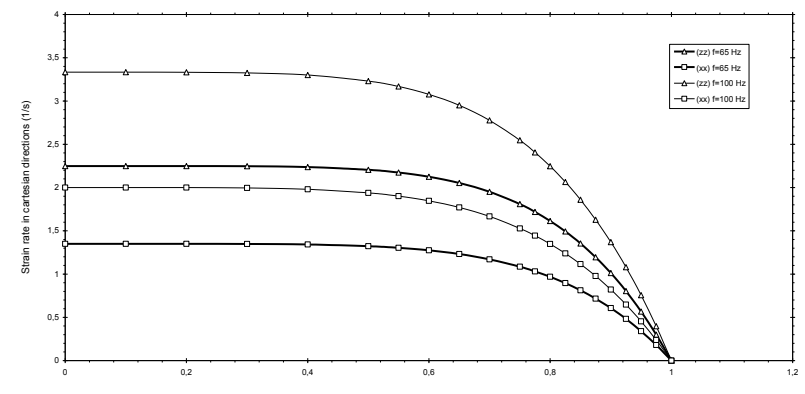

Fig. 5. Predicted HAZ strain rate distribution during linear friction welding of Ti 6Al 4V (amplitude of oscillation : $0.92 \mathrm{~mm}$, frequency of oscillation : $65 \mathrm{~Hz}$, friction pressure $25.9 \mathrm{MPa}$ )

\subsection{Analytical heat flow model}

For this phase an analytical two-dimensional model has been presented in literature [17]. The model assumes that specimens (Fig.6) are symmetric while the amplitude of oscillation is very small compared to the welding interface. It is also assumed that sliding velocity follows a square wave, as if both specimens oscillate sinusoidally in opposing directions producing a sliding speed that is always constant. Furthermore, the velocity of the material in the weld is made up of a component of the sliding velocity and a squeezing component due to normal pressure, with the sliding velocity being much greater than the squeezing velocity. Decoupling these two components leads to a steady state of temperature and a pseudo-steady state for sliding velocity, which is independent of time, a characteristic of an equilibrium phase. It is also assumed that at the centre of the weld, the highest temperatures are achieved and the material behaves in a fluid manner in a confined space. Due to its size it is treated as a lubricating layer. This assumption entails that inertia is negligible so that momentum equations reduce to stress balances, which is not the case as there are high accelerations in parts of the oscillatory cycle.

The main differences with lubrication theory are the constitutive equation modelling the rheology of the yielded alloy which is at a very high temperature, possibly near its melting point, and stress balances. Similarly to before, neglecting inertia the basic momentum equations are reduced to a squeezing and a sliding part of stress.

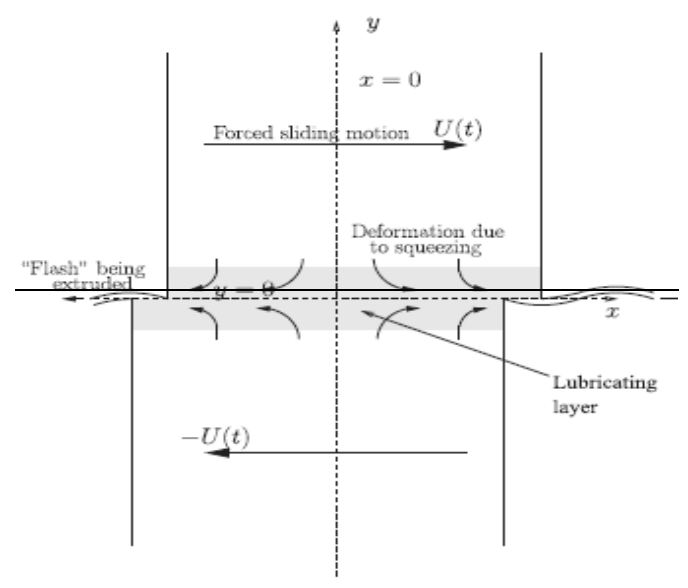

Fig. 6. Material flow model for extrusion phase [17]
Further to these momentum equations incompressibility applies and an energy equation determines temperature. The latter reduces to a simple linear convection-diffusion equation for the bulk of the specimen and an onedimensional diffusion equation for the very thin lubricating region at the interface. In the same work the material was assume to be hard so as to have an interface temperature very close to melting point.

In the thin soft layer at the interface the rates of shear differ by factors of order, and there is negligible heat loss from the specimen surface, making temperature and sliding velocity independent of $x$. As the steady state is considered, no heat losses are present from the specimen sides. The axial shortening rate is being dependent on friction pressure.

There were a number of observations deducted from this model such as that shear stress and approach velocity are both decreasing functions of sliding speed. The former is similar to the usual sliding friction being less than static friction.

One other key assumption in the developed model is that the size of the horizontal squeezing velocity is much smaller than the sliding velocity.

As no energy is lost by conduction or radiation, the power input is all used in warming the specimens explaining the fact that power input from sliding motion dominates that from squeezing motion.

When the material is considered to be softer, or that operating conditions are changed, so that the central (maximum) temperature is no longer close enough to $T \mathrm{~m}$ then stress appears to be a decreasing function of sliding speed. In the soft-material case, the maximum temperature depends on operating conditions.

In both material cases, a key dimensionless grouping was identified that needs to be small to use the key simplifying technique, decoupling variables into a dominant sliding and a smaller squeezing part.

\section{Conclusions}

Modelling of complex industrial processes such as linear friction welding can be addressed with the aid of various mathematical techniques to investigate individual process phases. These models may be based on experimental data for prediction or may provide qualitative information on the complex physical phenomena of this process.

During the initial frictioning phase conditioning of the welding interface is necessary for the correct development of a plastic material zone later in phase III. This can be studied with analytical contact models which can identify different frictional regimes and relate them to actual friction coefficient experiments. These will be used in process parameters selection, so as to promote the necessary conditions for welding, and numerical modelling of LFW. The use of ANNs for the same phase can assist in the prediction of the coefficient of friction, and have to be based on frictional behaviour experiments that associate parameters such normal pressure, rubbing velocity and interface temperature to friction coefficient and therefore to heat generation during phase I.

For the steady state extrusion phase of the process where plastic material flows into the upset while specimens shorten axially and in the end produce joints, different objectives through mathematical modelling. Analytical models of material velocity may predict the strain rates that material 
experiences at and near the welding interface. The value of strain rate directly influences localized yielding and subsequent plastic material flow. Unfavourable conditions of high strain rates for strain rate hardening materials may inhibit yield and will not produce conditions favourable to joining. Analytical models can also assist in the correct selection of process parameters by modeling material flow as a thin lubricating film.

\section{References}

1. P. Chapelle, N. Christakis, J. Wang, N. Strusevich, M.K. Patel, M. Cross, M., H. Abou-Chakra, J. Baxter, and U. Tüzün, P.I.Mech..Eng.E-J.Pro., 219, 43 (2005).

2. A. Vairis, and M. Frost, Mater. Mat. Sci.Eng. A-Struct,. A271, 477 (1999).

3. A. Vairis, Ph.D. thesis, University of Bristol, Bristol, 1998.

4. A.Vairis, Eur. J. Mech. A/ Solids, 16, 929 (1997).

5. A. Vairis, and N. Christakis, IJMIC , 2, 347 (2007).

6. A.E. Filippov, J. Klafter, and M. Urbakh, (2004), Phys. Rev. Lett., 92, 135503 (2004).

7. R. Budakian, and S.J. Putterman, Phys.Rev. Lett., 85, 1000 (2000). 8. J.Zhu, Y.Shi, X. Feng,, H. Wanga, and X. Lu, Materi. Design, 30, 1042 (2009)

9. S. Guessasma, M. Bounazef, and P. Nardin, Int.J Refract. Met. H, 24 240 (2006)

10. K. Genel , S.C. Kurnaz, and M. Durmanb, Mat. Sci.Eng. A-Struct,
A363, 203 (2003).

11. G. Zhanga, S. Guessasmaa, T.H. Liaoa, C. Coddeta, and J.M. Bordes, Surf. Coat. Tech., 2610 (2006).

12. Y. Karnavas and A. Vairis, Proc. IASTED-ASM, Heraklion (2011).

13. D.E. Rumelhart, and J.L. McClelland, Parallel distributed processing: explorations in the microstructure of cognition. Vol. 1: Foundations, MIT Press/Brandford Books, Cambridge, Massachusetts (1986).

14. K. Hornich, M. Stinchcomebe, and H. White, Neural networks, 2, 359 (1989).

15. K.S. Narendra, and K. Parthasarathy, IEEE T. Neural Networ., 1 , 4 (1990).

16. A. Vairis, and M. Frost, Mater. Mat. Sci.Eng. A-Struct,. 292, 8 (2000).

17. A.A Lacey, and C. Voong, Q.J . Mech Appl. Math., 65, 211 (2012). 